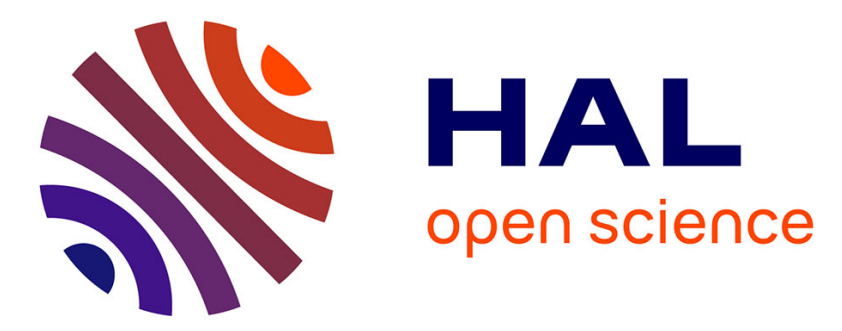

\title{
A Color Image Database for Haze Model and Dehazing Methods Evaluation
}

\author{
Jessica El Khoury, Jean-Baptiste Thomas, Alamin Mansouri
}

\section{To cite this version:}

Jessica El Khoury, Jean-Baptiste Thomas, Alamin Mansouri. A Color Image Database for Haze Model and Dehazing Methods Evaluation. 7th International Conference on Image and Signal Processing (ICISP 2016), May 2016, Trois-Rivières, Canada. pp.109-117, 10.1007/978-3-319-33618-3_12 . hal01343756

\section{HAL Id: hal-01343756 \\ https://hal.science/hal-01343756}

Submitted on 13 Jul 2016

HAL is a multi-disciplinary open access archive for the deposit and dissemination of scientific research documents, whether they are published or not. The documents may come from teaching and research institutions in France or abroad, or from public or private research centers.
L'archive ouverte pluridisciplinaire HAL, est destinée au dépôt et à la diffusion de documents scientifiques de niveau recherche, publiés ou non, émanant des établissements d'enseignement et de recherche français ou étrangers, des laboratoires publics ou privés. 


\title{
A color image database for haze model and dehazing methods evaluation
}

\author{
Jessica El Khoury, Jean-Baptiste Thomas, Alamin Mansouri \\ Le2i, Université de Bourgogne, \\ Bâtiment Mirande - UFR Sciences \& Techniques, \\ B.P. 47870 - 21078 Dijon Cedex, France. \\ jessica.el-khoury@u-bourgogne.fr
}

\begin{abstract}
One of the major issues related to dehazing methods (single or multiple image based) evaluation is the absence of the haze-free image (ground-truth). This is also a problem when it concerns the validation of Koschmieder model or its subsequent dehazing methods. To overcome this problem, we created a database called CHIC (Color Hazy Image for Comparison), consisting of two scenes in controlled environment. In addition to the haze-free image, we provide 9 images of different fog densities. Moreover, for each scene, we provide a number of parameters such as local scene depth, distance from the camera of known objects such as Macbeth Color Checkers, their radiance, and the haze level through transmittance. All of these features allow the possibility to evaluate and compare between dehazing methods by using full-reference image quality metrics regarding the haze-free image, and also to evaluate the accuracy of the Koschmieder hazy image formation model.
\end{abstract}

\section{Introduction}

When taking a picture in presence of dust, smoke or water particles hanging in the air, the light emanating from the scene and reaching the camera's sensor is scattered and attenuated. Similarly, the light coming from the light source is scattered by these particles, thus forming the so-called airlight [11] and resulting in an undesirable veil that reduces contrast and chroma in the picture [12. In this context, the image formation can be modeled as the sum of the scene's radiance and the airlight, weighted by a transmission factor $t(x)$ as in Equation 1.

$$
I(x)=J(x) t(x)+A_{\infty}(1-t(x))
$$

where $x$ denotes the pixel location. $I(x)$ is the image formed on the camera's sensor. $J(x)$ is the scene radiance. The transmission factor $t(x)$ depends on scene depth $d$ (distance to the sensor) and on the scattering coefficient $\beta$ of the haze, such that $t(x)=e^{-\beta \cdot d(x)}$ [11. Unlike more traditional image degradations, haze is a natural, depth-dependent noise that spans non-uniformly over the whole image. The degradation and the loss of information increases with depth, as the 
amount of fog between the imaged surface and the sensor increases. Hazy and foggy images have also different prevailing color, which depends on the scattering particles density and the ambient light [15]. The process to recover $I(x)$ from $J(x)$ by estimating first, the atmospheric light $A_{\infty}$ and $t(x)$, is known as dehazing or defogging.

Many dehazing methods have been proposed and compared to each other. Although, there exist a variety of dehazing algorithms for color images, not a single of them is usually accepted to work perfectly neither to work good enough. In addition, the resulting output of these algorithms is highly correlated to the concentration of scattering particles 7 .

Image dehazing and its quality evaluation remain difficult processes. Image dehazing is a transdisciplinary challenge, as it requires knowledge from different fields: meteorology to model the fog, optical physics to understand how light is affected by this fog and computer vision, as well as image and signal processing to recover the parameters of the scene. Image quality assessment (IQA) of dehazed images may requires the hazy and the haze-free images of the same real scene to use full-reference image quality metrics, which are captured under the same conditions such as illuminant, viewing geometry and resolution. Existing databases do not meet this feature (cf. Section 2). For this reason, we introduce in section 3 an original new color image database devoted to both haze model assessment and dehazing methods evaluation. We then discuss, in section 4, the limitations to invert the haze model without considering the physical aspect of hazy image formation before to conclude.

\section{Existing hazy image databases}

Several hazy image databases may be used for dehazing investigation. Hazy images databases including the haze-free reference image and other databases without reference. In the first category we find FRIDA (Foggy Road Image DAtabase) [18, 10] and FRIDA2 [17, 10, that represent evaluation databases for visibility and contrast restoration algorithms. These databases comprise a number of synthetic images with reference of urban road scenes and diverse road scenes, respectively. The view point is close to the one of the vehicle's driver. The software $S i V I C^{T M}$ was used to build physically-based road environments from a realistic complex urban model and to generate a moving vehicle with a physically-driven model of its dynamic behavior, and virtual embedded sensors. To each image without fog, four foggy images and a depth map are associated. The depth map is required to be able to add fog consistently in the images. Different kinds of fog are added to each of the four associated images: uniform fog, heterogeneous fog, cloudy fog, and cloudy heterogeneous fog. These four types of fog were inserted by applying the Koschmieder's law [1] by weighting differently the attenuation coefficient and/or the atmospheric light with respect to the pixel position. Despite the different aspects that are addressed in this database, simulated images fail to represent accurately the natural phenomena effects [8]. The physical interaction of light with atmospheric particles modifies 
the perceived colors, while colors in the simulated image maintain their hue information and only their saturation component shifts between the original color (saturated), and the haze color (unsaturated).

WILD (Weather and Illumination Database) [14, 6] is an outdoor urban scene database, acquired every hour over seasons. These images are taken under different weather and illumination conditions. Atmospheric conditions, scene distances and temporal data are also associated to images. First, this database cannot be used to compare one dehazed image with a reference due to the variation of illuminant. Second, small changes that could occur would bias a pixel by pixel comparison. Therefore, it appears important to us to build a new database, which does not suffer from these problems and more adequate for such evaluation.

On the other hand, developers often use hazy images of natural scenes, with no reference, which have usually a small size to evaluate dehazing methods within a short time using the minimum amount of resources [2, 5. However, the lack of the haze-free reference image makes the evaluation challenging and less reliable.

\section{Proposed database}

In the CHIC database [1], we consider two indoor scenes, Scene A and Scene B. Scenes were set up in a closed rectangular room, which is large enough to simulate the effect of the distance and the fog density on the objects radiance (length $=6.35 \mathrm{~m}$, width $=6.29 \mathrm{~m}$, height $=3.20 \mathrm{~m}$ ) with a large window (length $=5,54 \mathrm{~m}$, width $=1.5 \mathrm{~m}$ ) that allows a large amount of omnidirectional outdoor light to get in. It laterally covers the camera and the scenes. The photo session of each scene lasted 20 minutes. During this limited time, daylight is assumed to remain steady. This experiment was set up on February, from 1:00 to 4:00 p.m. when sunlight was not directly coming in through the window. Thus, the global light is close to the airlight in a cloudy day. Five Macbeth Color Checkers (MCCs) are placed in the scene at different distances to the camera. The farthest one serves to estimate the atmospheric light. This can be useful to follow up the color alteration when haze covers a scene. The scenes components present various colored surfaces types (reflective and glossy surfaces, rough surfaces, etc.).

A fog machine (FOGBURST 1500), which emits a dense vapor that appears similar to fog was used. A large quantity of fog is initially emitted until it is evenly distributed in the room and forms an opaque layer. Fog is then progressively evacuated through the window. This machine operates by evaporation. The vaporization of therein water-based liquid mixed with glycol, is done by heating. The particles of the ejected fog are water droplets, which have approximately the same radius size of the atmospheric fog $(1-10 \mu \mathrm{m})$.

Thereby, in addition to the haze-free image, nine images of different fog levels images of different levels of fog are captured (Table 1), from level 1, the highest fog density, to level 9, the lowest density. This set of images are captured under two illuminants: outdoor daylight and a compound light (outdoor light + ceiling lamp light (fluorescent tube)). For each level, an RGB image was acquired using 


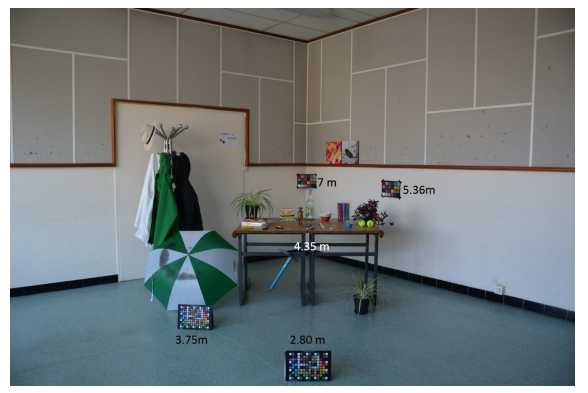

(a)

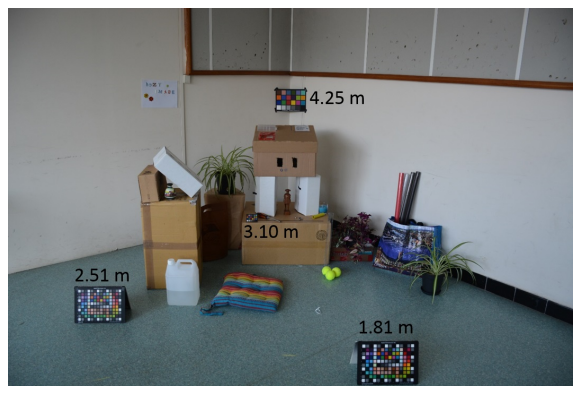

(b)

Fig. 1: Scene A (a) and Scene B (b). The distances of different MCCs to the camera are shown. The resolution of the image is $6000 \times 4000$.

the color camera Nikon D7100 4] providing NEF (RAW) and JPEG $6000 \times$ 4000 images. We used also the spectroradiometer Konica Minolta CS-2000 3 to measure the transmission of the fog on a white patch of the MCC placed at the back of the scene for each fog density, perpendicularly to the optical path. It was calibrated focusing on the same patch without fog.

\subsection{Scenes}

- Scene A: The shoot session of this scene was performed around 2:00 p.m. This scene shows a typical indoor view. We put on the table that is placed in the middle, a number of items with different characteristics such as shapes, colors, positions, surface types (glossy or rough surfaces) and textures. The wall behind the scene is half white and the top half with the white lines and the black holes represent distinctive elements to study algorithms handling near edges.

- Scene B: The shoot session of scene B was done two hours later. During this time the temperature of the illuminant significantly changed. Unlike the first one, the distance from the camera to the farthest point is smaller. It contains bigger geometric shapes. The fog densities which are randomly determined are characterized by the transmittance spectrum of each fog level.

The camera stayed still over the shoot session of each scene. However, the illuminant is not the same for both scenes, nor the distance to the camera and the density of fog for the correspondent levels (Table 1).

In Table 1, the relative transmittance of fog is calculated with respect to airlight at a given distance over the black patch of original hazy images, as follows:

$$
T=1-\frac{S_{\text {levelx }}-S_{\text {airlight }}}{S_{\text {haze-free }}-S_{\text {airlight }}}
$$


Where $S_{\text {levelx }}, S_{\text {airlight }}$ and $S_{\text {haze-free }}$ are the spectral values of green in images of different fog levels, of the airlight image of our database where the scene is completely covered by fog and the haze-free image.

Table 1: Relative transmittance $\mathrm{T}$ of fog in original hazy images of scene $\mathrm{A}$ and scene B. Level 1: highest fog density. Level 9: lowest fog density.

\begin{tabular}{|l|c|c|c|c|c|c|c|r|r|}
\hline T & Level 1 & Level 2 & Level 3 & Level 4 & Level 5 & Level 6 & Level 7 & Level 8 & Level 9 \\
\hline Scene A & $100 \%$ & $92 \%$ & $90 \%$ & $91 \%$ & $84 \%$ & $71 \%$ & $75 \%$ & $52 \%$ & $28 \%$ \\
\hline Scene B & $100 \%$ & $97 \%$ & $89 \%$ & $91 \%$ & $74 \%$ & $64 \%$ & $55 \%$ & $30 \%$ & $15 \%$ \\
\hline
\end{tabular}

Comparing to outdoor scenes, the light source is located at infinity and the particles forming the synthetic fog are water particles fall in the same range of particles' size, therefore the same scattering law is applied. Since the room where the shoot session was running is not an open place, the airlight color seems to be close to dark gray. According to Figure 2, the calculated values of $A$ and $t$ are almost the same over channels at all fog levels. All of these facts prove that, the outside conditions are almost fulfilled in our database.

\section{Limitation of Koschmieder model for image recovery}

Single image dehazing methods, which consider the haze model given in Equation 1. are usually based on strong assumptions to estimate $A_{\infty}$ and $t(x)$ from the single RGB hazy image [9, 16. However, the only way to verify these assumptions and the model as well, is to have the haze-free image.

Consequently, $A_{\infty}$ is the $\mathrm{R}, \mathrm{G}$ and $\mathrm{B}$ values of the fog layer calculated from image level 1, which is uniformly covered by fog. For each level, the airlight $A=A_{\infty}(1-t(x))$ is similarly calculated over the black patch of MCC placed at the scene's back (color patch within the red surrounding in Figures 3 and 4 ). We subtracted from it the offset values of R, G, and B of a patch of $20 \times 20$ pixels of the same black patch from the original image without fog.

Once $A_{\infty}$ and $A$ are estimated, $t(x)$ of a given scene depth is deduced from the second part of equation 1 over the same color chart. Since the distance of each chart is known, the scattering coefficient is deduced and the transmission matrix is then calculated over the entire image, using approximative depths of secondary objects. Based on the known distances of MCCs, images have been split into four zones. Particular focus should be given to the color patch within the red surrounding, which has an accurate known distance to the camera, and has also the lowest object's transmission.

For the sake of simplicity and due to space constraints, one out of two adjacent levels of daylight images, is retained for image recovery study (Figures 3 and 4 . 

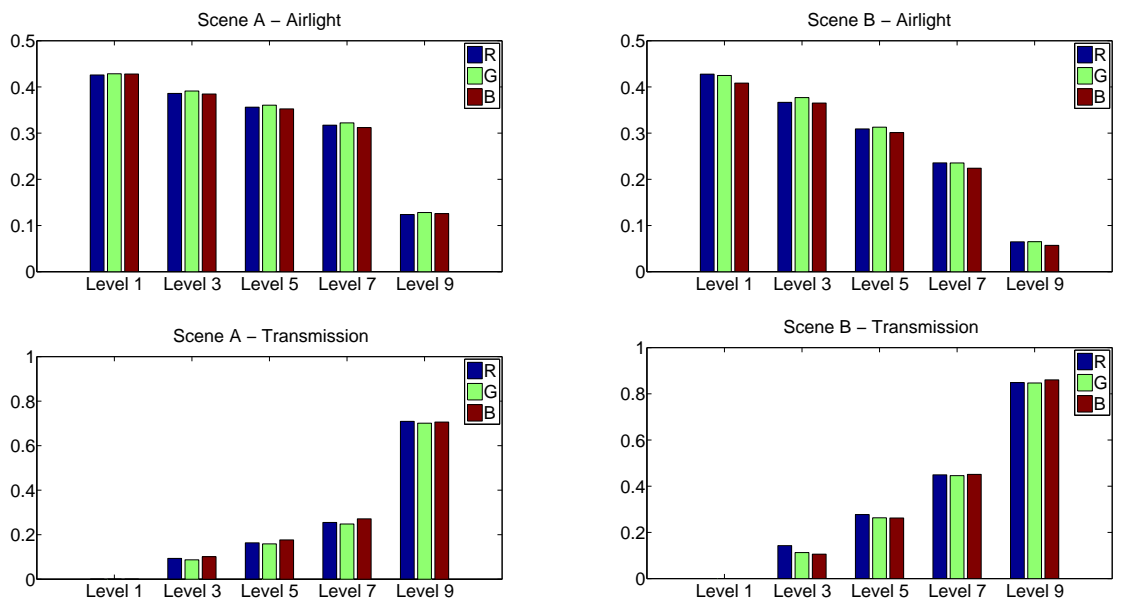

Fig. 2: Estimated $A$ and $t$ obtained for each level of haze for a given distance of the MCC placed at the back of the scene. First row: airlight, second row: transmission. First column: scene A, second column: scene B.

Although Scene A and Scene B differ slightly from each other by depths and fog densities, corresponding images of both scenes of the same level provide close values of $A$ and $t$. This gets reflected through reconstructed images (Figures 3 and 4), where the area within the red surrounding is poorly recovered in level 1 and level 3 from $t_{1}=0.00$ to $t_{2}=0.09$ in Scene $\mathrm{A}$ and from $t_{1}=0.00$ to $t_{2}=0.12$ in Scene B, respectively. From level 5 and on up, when $t$ exceeds 0.17 in Scene A and 0.27 in Scene B, the inversion of the haze model (equation 1) succeeds to recover recognizable features comparing to the haze-free image. Since unknown parameters of all fog levels are similarly estimated, this means that the induced error is the same. When the fog density is relatively high (in our case, below level 5), even if the estimation is reasonably accurate, it seems difficult, if not impossible, to compensate thanks to it the lost transmitted light through scattering and absorption and getting a reconstructed image close to the hazefree image features. This is clearly shown on images of low levels of fog, where reconstructed images are noticeably noisy with perceptible saturation shift. This comes to confirm once more what has been pointed out by Narasimhan and Nayar [13], that this model is not valid for depths that are more than a few kilometers. Similarly, when the amount of fog greatly increases, even the radiance of near objects is no more well captured by the camera. Moreover, since the exponential attenuation comes quickly down to zero, the noise is greatly amplified for high fog densities. 


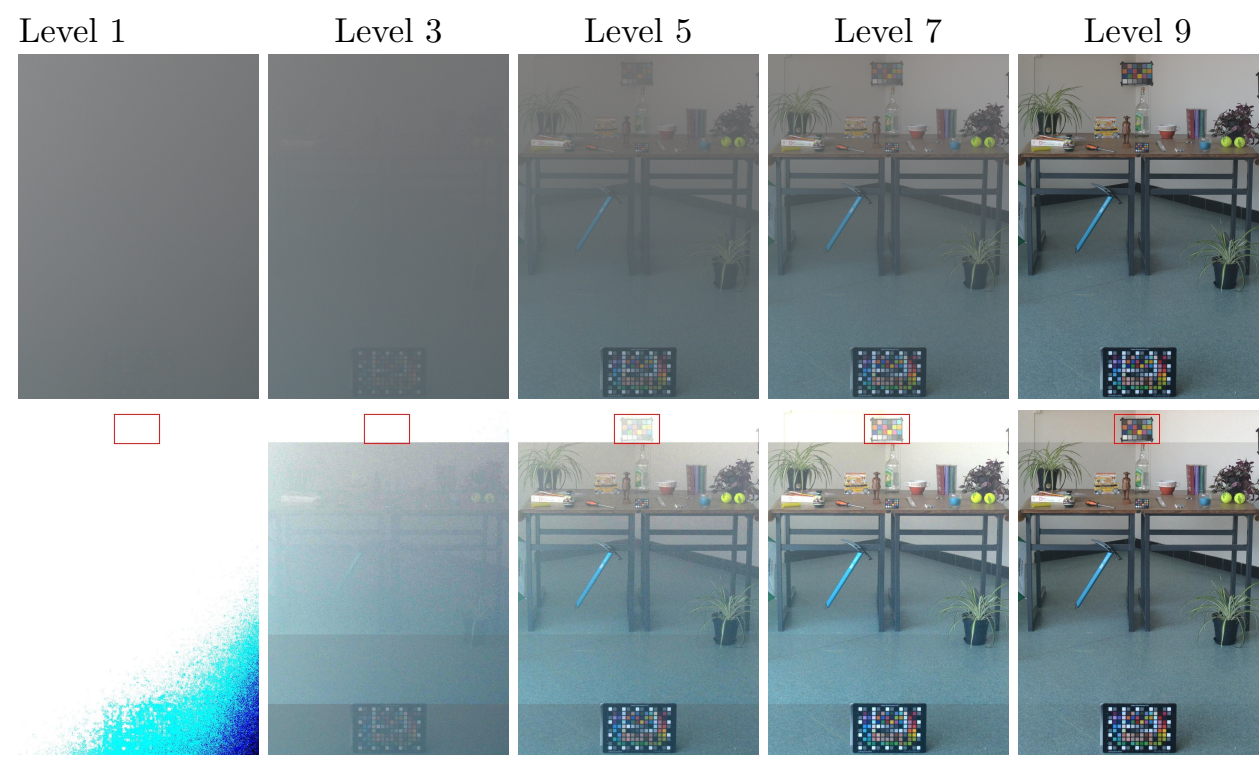

Fig. 3: The original hazy (first row) and the reconstructed images of Scene A (second row). $A$ and $t$ are estimated on the surrounded red color patch. The distance between it and the camera is $7 \mathrm{~m}$. First row: original hazy images, second row: reconstructed images. Cropped image size is $1537 \times 2049$.

\section{Conclusion and future work}

We proposed and described a new color hazy image database of two indoor static scenes. The haze-free image and the supplementary data help to evaluate the commonly accepted haze model, and to evaluate and compare dehazing methods. Such evaluation is done when we consider how much a dehazed image processed by a given method succeeds to meet haze-free image. No matter what circumstances lead up to visibility degradation. Although the parameters of the haze model are accurately estimated, from a level of data lost, when the transmission of the scene is very low, the haze-free image is no longer properly recovered. Koschmieder's model is therefore considered to be not valid for high densities of fog.

Our future work will use this database for an objective assessment of several dehazing methods using IQA indices including full-reference metrics. We will study also the correlation of these metrics with the perceptual judgments as an efficient attempt to develop a proper haze-dedicated evaluating metric. 


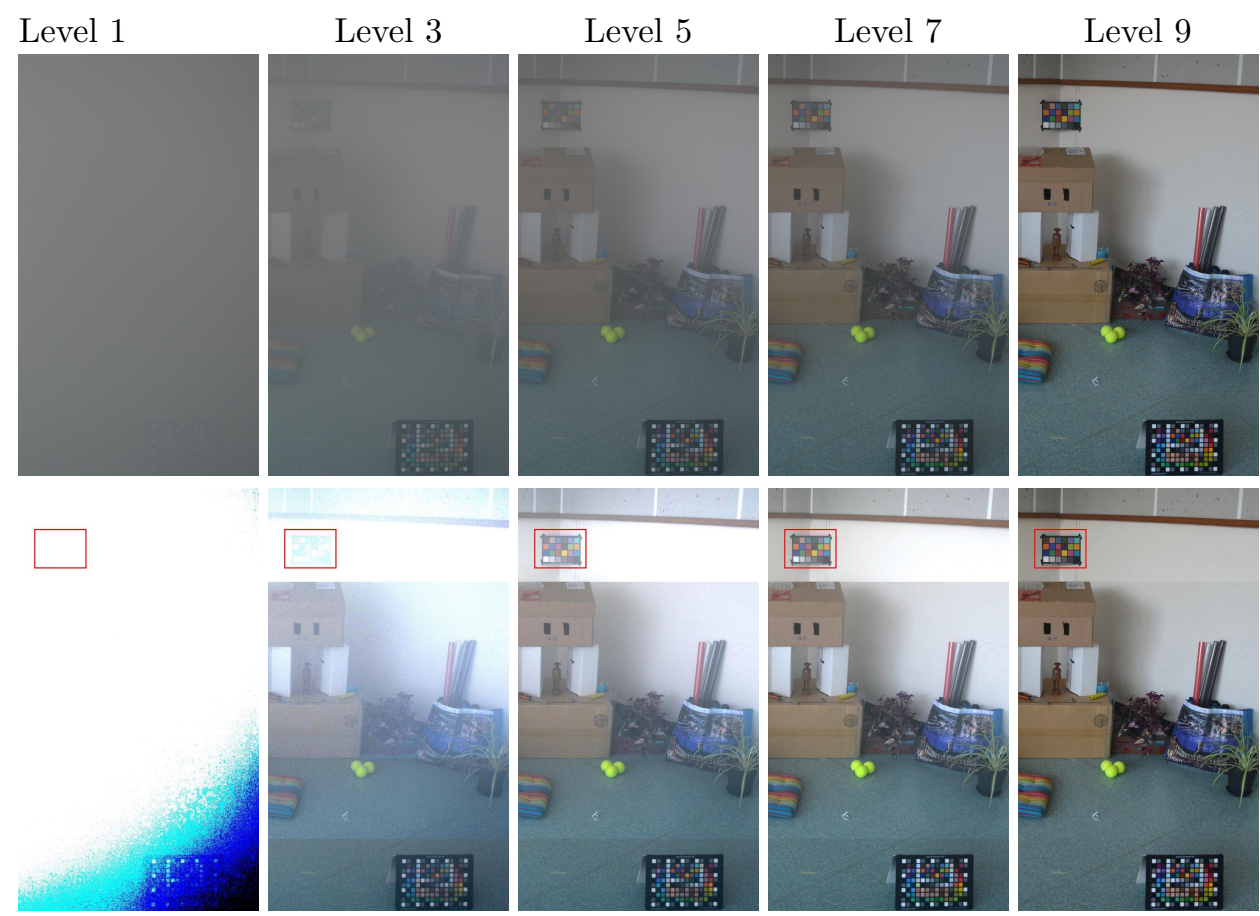

Fig. 4: The original hazy (first row) and the reconstructed images of Scene B (second row). $A$ and $t$ are estimated on the surrounded red color patch. The distance between it and the camera is $4.25 \mathrm{~m}$. First row: original hazy images, second row: reconstructed images. Cropped image size is $1537 \times 3073$.

\section{Acknowledgment}

The authors thanks the Open Food System project for funding. This project is a part of The Investments for the Future Programme managed by Bpifrance, www.openfoodsystem.fr.

The final publication is available at Springer via http://dx.doi.org/10. 1007/978-3-319-33618-3_12 


\section{Bibliography}

[1] CHIC (Color Hazy Image for Comparison). http://chic.u-bourgogne.fr.

[2] Image dehazing. http://ivrl.epfl.ch/supplementary_material/SFS_ ICIP09. Accessed March 1, 2016.

[3] Konica minolta, cs-2000 spectroradiometer. http://sensing. konicaminolta.us/products/cs-2000-spectroradiometer/. Accessed April 1, 2015.

[4] User's manual, nikon d7100. http://cdn-10.nikoncdn.com/pdf/ manuals/dslr/D7100EN.pdf. Accessed April 1, 2015.

[5] Waterloo ivc dehazed image database. http://ivc.uwaterloo.ca/ database/Dehaze/Dehaze-Database.php. Accessed March 1, 2016.

[6] Wild (weather and illumination database). http://www.cs.columbia. edu/CAVE/software/wild/index.php. Accessed April 1, 2015.

[7] Jessica El Khoury, Jean-Baptiste Thomas, and Alamin Mansouri. Does dehazing model preserve color information? In Signal-Image Technology and Internet-Based Systems (SITIS), 2014 Tenth International Conference on, pages 606-613. IEEE, 2014.

[8] Jessica El Khoury, Jean-Baptiste Thomas, and Alamin Mansouri. Haze and convergence models: Experimental comparison. In AIC 2015, 2015.

[9] Kaiming He, Jian Sun, and Xiaoou Tang. Single image haze removal using dark channel prior. Pattern Analysis and Machine Intelligence, IEEE Transactions on, 33(12):2341-2353, 2011.

[10] IFSTTAR. Frida (foggy road image database). $\quad$ http://www.sciweavers.org/read/ frida-foggy-road-imagedatabase-evaluation-database-for $\backslash$ /-visibility-restoration-algorithms-184350/. Accessed April $1,2015$.

[11] Harald Koschmieder. Theorie der horizontalen Sichtweite: Kontrast und Sichtweite. Keim \& Nemnich, 1925.

[12] Srinivasa G Narasimhan and Shree K Nayar. Chromatic framework for vision in bad weather. In Computer Vision and Pattern Recognition, 2000. Proceedings. IEEE Conference on, volume 1, pages 598-605. IEEE, 2000.

[13] Srinivasa G Narasimhan and Shree K Nayar. Contrast restoration of weather degraded images. Pattern Analysis and Machine Intelligence, IEEE Transactions on, 25(6):713-724, 2003.

[14] Srinivasa G Narasimhan, Chi Wang, and Shree K Nayar. All the images of an outdoor scene. In Computer Vision-ECCV 2002, pages 148-162. Springer, 2002.

[15] Shree K Nayar and Srinivasa G Narasimhan. Vision in bad weather. In Computer Vision, 1999. The Proceedings of the Seventh IEEE International Conference on, volume 2, pages 820-827. IEEE, 1999. 
[16] Jean-Philippe Tarel and Nicolas Hautière. Fast visibility restoration from a single color or gray level image. In Computer Vision, 2009 IEEE 12th International Conference on, pages 2201-2208. IEEE, 2009.

[17] Jean-Philippe Tarel, Nicolas Hautière, Laurent Caraffa, Aurélien Cord, Houssam Halmaoui, and Dominique Gruyer. Vision enhancement in homogeneous and heterogeneous fog. Intelligent Transportation Systems Magazine, IEEE, 4(2):6-20, 2012.

[18] Jean-Philippe Tarel, Nicolas Hautière, Aurélien Cord, Dominique Gruyer, and Houssam Halmaoui. Improved visibility of road scene images under heterogeneous fog. In Intelligent Vehicles Symposium (IV), 2010 IEEE, pages 478-485. IEEE, 2010. 